\title{
Long-Term Follow-Up of
} Intravitreal Ranibizumab for the Treatment of Choroidal Neovascularization due to Choroidal Osteoma

\author{
Zenith H.Y. Wu Michelle Y.Y. Wong Timothy Y.Y. Lai \\ Department of Ophthalmology and Visual Sciences, The Chinese University of \\ Hong Kong, Hong Kong, SAR, China
}

\section{Key Words}

Choroidal osteoma $\cdot$ Choroidal neovascularization $\cdot$ Ranibizumab

\begin{abstract}
Choroidal osteoma is an uncommon benign osseous intraocular tumor that typically affects young adult women. Choroidal neovascularization (CNV) is one of the complications that can develop in eyes with choroidal osteoma. We present a case of CNV secondary to choroidal osteoma treated with intravitreal ranibizumab. A 57-year-old lady presented with painless loss of vision with a right-eye visual acuity of 20/800. Fundus examination showed a welldemarcated yellowish peripapillary choroidal osteoma with associated retinal and subretinal hemorrhage due to CNV. Three intravitreal ranibizumab injections at monthly intervals were given and her visual acuity improved to 20/30 following treatment. After 1.2 years of followup, the right eye visual acuity was maintained at 20/30 with no evidence of CNV recurrence. Our findings suggest that intravitreal ranibizumab may be an effective therapeutic option for treating CNV secondary to choroidal osteoma.
\end{abstract}

\section{Introduction}

Choroidal osteoma is a rare ossifying benign tumor of the choroid [1]. It was first described by Gass et al. in 1978, when they reported the development of yellowish, slightly elevated juxtapapillary choroidal lesions with sharp geographic borders in four healthy young women [1]. The tumor is usually unilateral and develops in the second or third decade of life. Long-term longitudinal studies have shown that $50 \%$ of patients with choroidal osteoma had a final vision of $20 / 200$ or worse [2,3]. Tumor growth, tumor decalcification, and the development of choroidal neovascularization (CNV) can cause substantial visual loss [2]. Thermal laser photocoagulation [4], transpupillary 
thermotherapy [5], photodynamic therapy (PDT) with verteporfin [6], and more recently intravitreal anti-vascular endothelial growth factor (anti-VEGF) therapy like bevacizumab [7-9] have been reported as treatment options of CNV secondary to choroidal osteoma with variable results. There has only been one previous publication of the use of intravitreal ranibizumab for the treatment of CNV due to choroidal osteoma [10]. However, the patient was only followed for 6 months and the long-term outcome was not available. We hereby report a patient with CNV secondary to choroidal osteoma treated with intravitreal ranibizumab with long-term follow-up.

\section{Case Report}

A 57-year-old lady presented with right-eye painless visual loss for 10 days. Her past ocular and medical histories were unremarkable. On presentation, the visual acuity was 20/800 in the right eye. Fundus examination of the right eye revealed a large well-demarcated yellowish peripapillary choroidal lesion of 10-disc area with associated retinal and subretinal hemorrhage involving the central macula (fig. 1a). Fluorescein angiography (FA) of the right eye showed subfoveal CNV with late leakage (fig. 1b and c). A diagnosis of right eye CNV secondary to choroidal osteoma was made. After discussing the treatment options with the patient, she underwent intravitreal injections of $0.5 \mathrm{mg}$ ranibizumab (Lucentis, Novartis Inc., Basel, Switzerland). Three loading doses at monthly intervals were given. Following the three ranibizumab injections, the retinal and subretinal hemorrhage completely absorbed (fig. 2a) and the right eye visual acuity improved to 20/30. Repeat FA at 3 months demonstrated fibrosis of the CNV with absence of fluorescein leakage (fig. $2 \mathrm{~b}$ and $\mathrm{c}$ ). The patient was followed for 1.2 years after presentation and her BCVA remained stable at 20/30 with no recurrence of CNV.

\section{Discussion}

Choroidal osteoma is an unusual intraocular tumor of unknown etiology and one of the most important causes of visual loss in patients with choroidal osteoma is the development of CNV [2,3]. Shields et al. [2] reported the long-term outcome of 61 patients with choroidal osteoma and it was shown that patients with choroidal osteoma have a $31 \%$ chance of developing CNV by 10 years. A similar study by Aylward et al. [3] demonstrated that $47 \%$ of 36 patients with choroidal osteoma developed CNV. Thermal laser photocoagulation has been described as a treatment option for CNV secondary to choroidal osteoma but with limited success [4]. The poor outcome of thermal laser photocoagulation was speculated to be due to lack of pigmentation within the tumor, as well as due to the thin and degenerated retinal pigment epithelium overlying the tumor resulting in unsatisfactory laser reaction [4]. PDT with verteporfin and transpupillary thermotherapy have also been described as treatment options for CNV secondary to choroidal osteoma, but reports on favorable treatment outcomes were scarce $[5,6]$.

More recently, intravitreal injections of anti-VEGF agents such as bevacizumab and ranibizumab were described as possible interventions for CNV secondary to choroidal osteoma [7-10]. This was inspired by the safe and effective use of intravitreal antiVEGF antibodies in treating CNV in cases of neovascular age-related macular degeneration as well as other types of CNV. Song and Roh [10] first reported the use of intravitreal ranibizumab in a patient with CNV secondary to choroidal osteoma and observed regression of $\mathrm{CNV}$ as well as recovery of visual acuity. In our case, there was considerable visual improvement from 20/800 to 20/30 after three loading doses of intravitreal ranibizumab injections. Moreover, there was no evidence of CNV recurrence 15 months after initial treatment. Our case further supports the role of intravitreal ranibizumab in the treatment of CNV secondary to choroidal osteoma. 

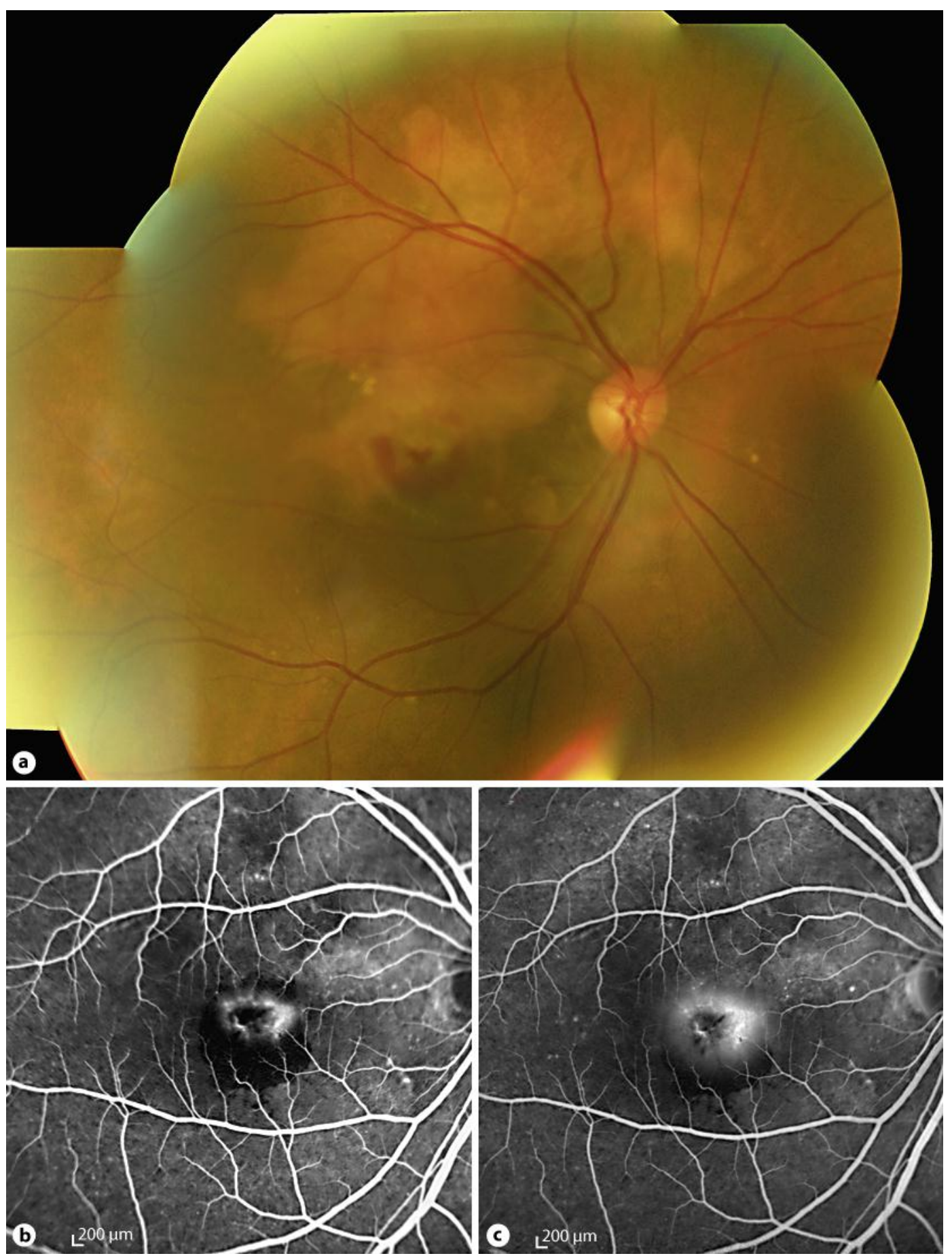

Fig. 1. a Pretreatment mosaic fundus photograph of the right eye showing well-demarcated yellowish white choroidal osteoma with retinal and subretinal hemorrhage at central macula. b Early- and c late-phase fluorescein angiography showing a subfoveal hyperfluorescent lesion due to choroidal vascularization associated with blocked fluorescence due to hemorrhage. 

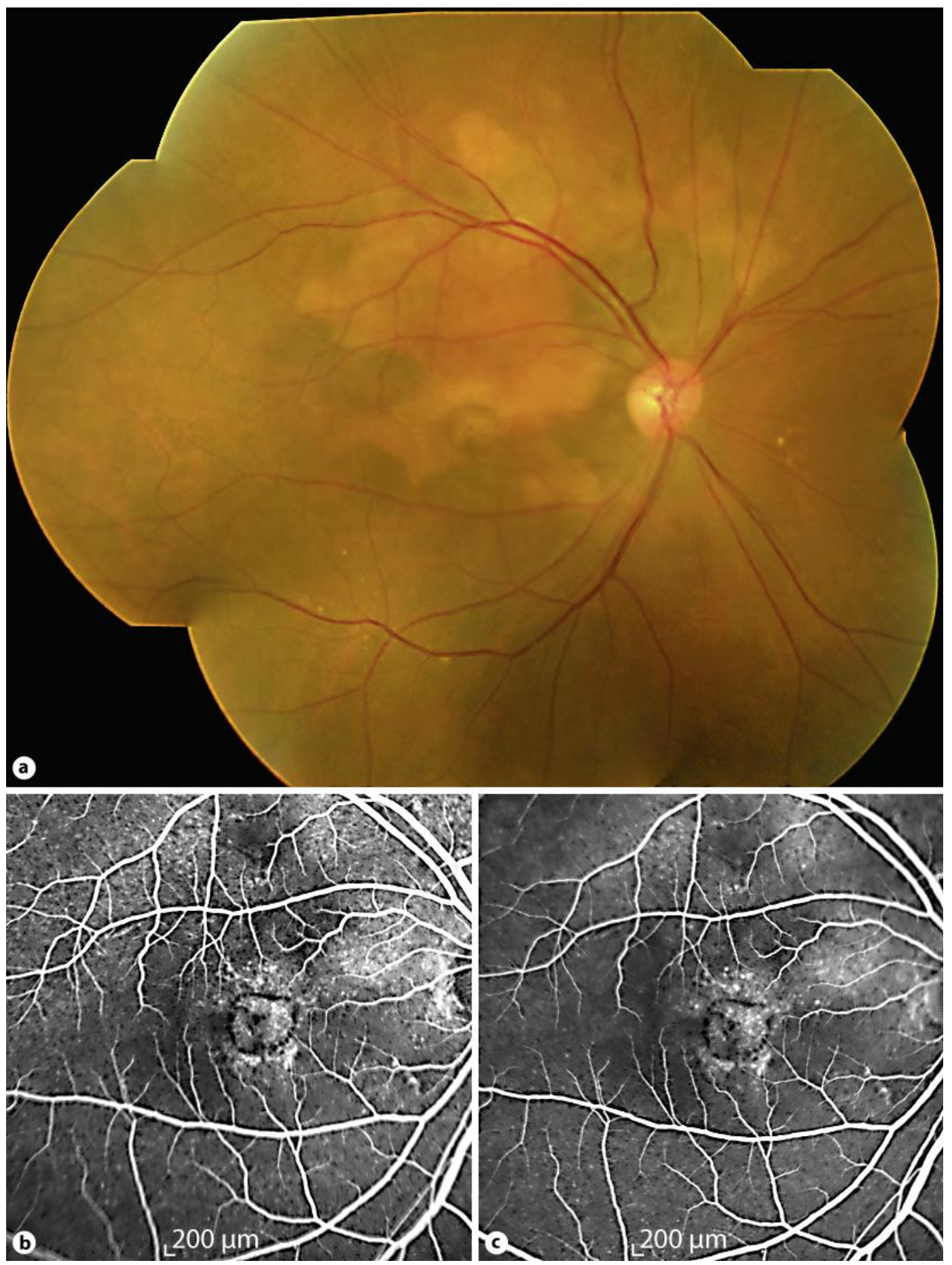

Fig. 2. a Mosaic fundus photograph of the right eye at 3 months following intravitreal ranibizumab treatment showing complete resolution of macular hemorrhage with mild fibrosis at the inferior border of the choroidal osteoma. $\mathbf{b}$ Early- and $\mathbf{c}$ late-phase fluorescein angiography showing fibrosis of the choroidal neovascularization with absence of leakage. 


\section{References}

1 Gass JD, Guerry RK, Jack RL, Harris G: Choroidal osteoma. Arch Ophthalmol 1978;96:428-435

$\checkmark 2$ Shields CL, Sun H, Demirci H, Shields JA: Factors predictive of tumor growth, tumor decalcification, choroidal neovascularization, and visual outcome in 74 eyes with choroidal osteoma. Arch Ophthalmol 2005;123:1658-1666.

-3 Aylward GW, Chang TS, Pautler SE, Gass JD: A long-term follow-up of choroidal osteoma. Arch Ophthalmol 1998;116:1337-1341.

4 Rose SJ, Burke JF, Brockhurst RJ: Argon laser photoablation of a choroidal osteoma. Retina 1991;11:224228.

5 Shukla D, Tanawade RG, Ramasamy K: Transpupillary thermotherapy for subfoveal choroidal neovascular membrane in choroidal osteoma. Eye (Lond) 2006;20:845-847.

-6 Shields CL, Materin MA, Mehta S, Foxman BT, Shields JA: Regression of extrafoveal choroidal osteoma following photodynamic therapy. Arch Ophthalmol 2008;126:135-137.

7 Ahmadieh H, Vafi N: Dramatic response of choroidal neovascularization associated with choroida osteoma to the intravitreal injection of bevacizumab (Avastin). Graefes Arch Clin Exp Ophthalmol 2007;245:1731-1733.

>8 Song WK, Koh HJ, Kwon OW, Byeon SH, Lee SC: Intravitreal bevacizumab for choroidal neovascularization secondary to choroidal osteoma. Acta Ophthalmol 2009;87:100-101.

9 Pandey N, Guruprasad A: Choroidal osteoma with choroidal neovascular membrane: successful treatment with intravitreal bevacizumab. Clin Ophthalmol 2010;4:1081-1084.

10 Song MH, Roh YJ: Intravitreal ranibizumab in a patient with choroidal neovascularization secondary to choroidal osteoma. Eye (Lond) 2009;23:1745-1746. 